\title{
Comparison of waterbird population and their habitat utilization of two different reservoirs in Salingyi township, Sagaing region
}

\begin{abstract}
Waterbird population and habitat variables at two different reservoirs (Salingyi and Ngwetha) were compared in order to determine the habitat utilization of waterbird species from June 2017 to May 2018. A total of 29 waterbird species belonging 24 genera. 10 families and six orders were recorded through direct observation in both reservoirs (28 waterbird species in Salingyi reservoir (Site I) and 22 species in Ngwe-tha reservoir (Site II)). Out of 29 species, 20 species were residents and nine species were winter visitors. According to IUCN Red list, Near-threatened species of Black-headed Ibis Threskiornis melanocephalus was recorded in Salingyi reservoir .The population of Lesser Whistling-duck Dendrocygna javanica was highest number and was observed the marshy swamp and open water body of Salingyi reservoir. Common Coot Fulica atra was as the highest population and was dominant in the open water area in Ngwe-tha reservoir. According to foraging behavior, the higher population of waterbirds recorded in shorebirds (2667 birds) than dabbling ducks (1764 birds) and diving birds (1557 birds) at Site I. In Site II, the highest population of waterbirds recorded in diving birds (1625 birds) followed by dabbling ducks (641 birds) and shorebirds (578 birds). The results showed that the populations of waterbirds in Salingyi reservoir were significantly different from Ngwe-tha reservoir. This indicated that Salingyi reservoir may vary in different wetland habitats such as open water area, marshy swamp, flooded agricultural fields, shrub and bushy area, small grass land and small trees around the reservoir than Ngwe-tha reservoir, mostly composed of open water area and sparsely small trees and shrubs edge of open water area. This might be due to the presence of aquatic vegetative composition and vegetation structure, occurrence of suitable foraging and breeding sites that had attracted the highest number of species and population of waterbirds to utilize the Salingyi reservoir.
\end{abstract}

Keywords: reservoir, population of waterbird, near-threatened species, habitats utilization and foraging behavior
Volume 4 Issue 4 - 2019

\author{
Htay Khaing, Nay Lin Oo, Thin Thin Khaing \\ Monywa University, Myanmar
}

Correspondence: Htay Khaing, Monywa University, Myanmar, Email seintsaintwin22@gmail.com

Received: June 27, 2019 | Published: August 02, 2019

\section{Introduction}

The wetlands of Myanmar include rivers and streams, shallow fresh storage reservoirs, fish ponds, seasonal flooded cultivated plains, and estuaries with extensive mangrove swamps. The local people depend on wetland area for foods and water resources. Wetlands are one of the most productive ecosystems in the world. They provide important functions in erosion control, flood control, aquifer recharge and nutrient absorption. ${ }^{1}$ Wetlands are major breeding, nesting, and migration staging areas for waterfowls and shorebirds. ${ }^{2}$ Wetlands are fringe habitats between terrestrial and aquatic ecosystems. ${ }^{3}$

Wetlands are important water filters. They also provide habitats for large number of fauna and flora. The vast numbers of invertebrates such as worms and small shellfish contained in the mud provide food for internationally important populations of migratory waterbirds. ${ }^{4}$ Some waterbirds are more terrestrial or aquatic than others and their adaptations include webbed feet, bills and legs adapted to feed in water and the ability to dive from the surface or the air to catch prey in water. ${ }^{5}$ Many species of birds use different habitat at different time of the year. These habitat differences are due to the fact that non breeding habitats do not need to provide for nesting activities. Migratory species may be forced to use different habitats in different regions simply because of limited choice in availability. Nevertheless, migratory birds use similar habitats throughout the year. ${ }^{6}$
Habitat selection in birds may greatly vary from species to species, depending upon the morphology of the bill (i.e straight, elongated, slender, curved bills), prey availability (i.e richness and vulnerability to capture) and foraging behavior such as visual vs tactile foragers. ${ }^{7}$ The range of accessible water depths also depend on foraging behaviors. Compared to shorebirds, waterfowl can use diverse foraging behaviors (such as surface dabbling, tipping-up, and head-submerging), each of which is suitable for foraging at a different water depth. Salingyi and Ngwe-tha reservoirs are located in Salingyi Township, Sagaing Region. These reservoirs were constructed for water supply to paddy field and also such as a potential water body for fish production. The most of field around the reservoirs, the local people used for rice and other crop cultivation. Moreover, these reservoirs composed of different habitats such as shrubs, small trees, open water body, shallow water area, marshy swamp area, agricultural fields and other vegetation. The main objectives of this study were to determine and compare the waterbird population and their habitat utilization of Salingyi and Ngwe-tha reservoirs.

\section{Materials and methods}

\section{Study area}

Salingyi Township is located in Sagaing Region which lies between $21^{\circ} 53^{\prime} 35^{\prime \prime}$ to $22^{\circ} 01^{\prime} 52^{\prime \prime} \mathrm{N}$ and $95^{\circ} 04^{\prime} 34^{\prime \prime}$ to $95^{\circ} 56^{\prime} 02^{\prime \prime} \mathrm{E}$. It is bounded Chindwin River in East, Palae Township in West, Yaesagho 
and Myaing Township in South and Yinmabin Township in North Two different study sites were designated in Salingyi Township (Figure 1)

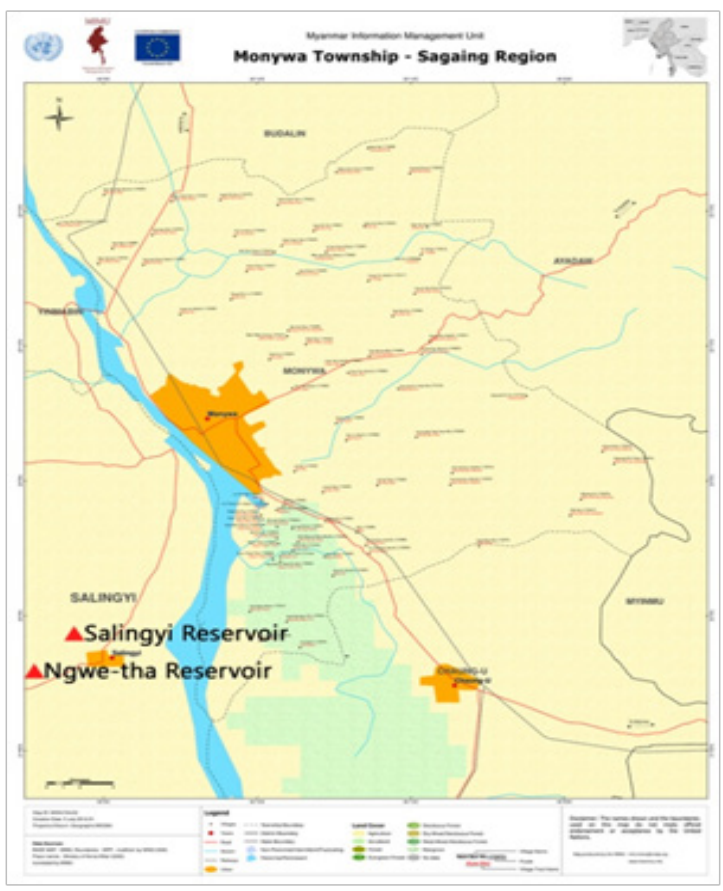

Figure I Location map of the study area (Source: MIMU, 2012).

\section{Site (I) Salingyi reservoir}

Salingyi reservoir is located near the western side of the Salingyi Township. It is situated between $21^{\circ} 58^{\prime} 11^{\prime \prime} \mathrm{N}$ to $21^{\circ} 59^{\prime} 00^{\prime \prime} \mathrm{N}$ and $95^{\circ} 03^{\prime} 11^{\prime \prime} \mathrm{E}$ to $95^{\circ} 03^{\prime} 54^{\prime \prime} \mathrm{E}$. The total surface area is about 97.3 hectares. The maximum water depth of the study area is about 5.18 $\mathrm{m}$ and the minimum water depth is $2.13 \mathrm{~m}$. This reservoir was open water area and composed of shallow water area, marshy swamp, damp soil, flooded agricultural fields, cultivated fields, shrub and bushy area, small grass land and small trees around the reservoir.

\section{Site (II) Ngwe-tha reservoir}

Ngwe-tha reservoir is located south western side of the Salingyi Township. It is situated between $21^{\circ} 53^{\prime} 53^{\prime \prime} \mathrm{N}$ to $21^{\circ} 55^{\prime} 28^{\prime \prime} \mathrm{N}$ and $95^{\circ} 01^{\prime} 49^{\prime \prime} \mathrm{E}$ to $95^{\circ} 02^{\prime} 03^{\prime \prime} \mathrm{E}$. The total surface area is about 200 hectares. The maximum water depth of the study area is about 5.18 $\mathrm{m}$ and the minimum water depth is $0.91 \mathrm{~m}$. This reservoir was mostly open water area and composed of edge of open water area and sparsely small trees and shrubs around the reservoir (Figure 1).

\section{Study period}

The present study was conducted from June, 2017 to May, 2018.

\section{Field technique}

The field survey was conducted twice per month. The birds photos were taken with digital camera (EOS 700D with Sigma 150-600 mm and Powershot SX530 HS). Bird watching was undertaken two times during the period from 6:30 am to 11:30 am in the morning and from 3:00 pm to $5: 30 \mathrm{pm}$ in the evening. Bird watching and counting were carried out using a boat and also walking to each study sites. Bird census was made by using the point count method employing direct observation use a binocular. At each sampling point, all birds seen or heard were recorded during 10 minutes. Census was not conducted during heavy rainy and cloudy days.

\section{Identification and classification}

The waterbird species were identified..$^{9-11}$ Birds were listed in the classification systems proposed by Bird Life international checklist. ${ }^{12}$

\section{Foraging behavior}

Foraging behavior of the waterbird species was recorded on direct observations during every field surveys. The waterbirds were grouped based on foraging behavior and habitats use such as dabbling duck (small or middle size of Anatidae species that require shallow wetland for feeding), diving waterbird (includes all kinds of waterbird species that can dive for feeding) and shorebird (wader species and are not good at swimming) according to Chan-Woo et al., ${ }^{13}$ and based on visual observations.

\section{Analysis of data}

Comparison using analysis of variance (ANOVA) test to determine the significant difference of waterbird population between the two different reservoirs, was also evaluated using (SPSS version 23).

\section{Results}

A total of 29 waterbird species belonging 24 genera, 10 families and six orders were recorded in both reservoirs that is Salingyi reservoir and Ngwe-tha reservoir (Table 1). Out of 29 waterbird species, 28 species were recorded in Salingyi reservoir and 22 species from Ngwetha reservoir. The total of 17 species were commonly observed in both reservoirs but six species of winter visitor were absent in Ngwe-tha reservoir and the resident species, Great Crested Grebe (Podiceps cristatus ) was absent in Salingyi reservoir. The near-threatened species of Black-headed Ibis (Threskiornis melanocephalus) was recorded in Salingyi reservoir (Table 1).

\section{Comparison of waterbird population of two different reserviors}

The total numbers of water birds (5988 birds) were recorded in Salingyi reservoir (Site I). Bird numbers reached a peak in December (754 birds), January (875 birds) and February ( 833 birds) and gradually decline as birds left the reservoir in May (294 birds) and June (266 birds). The highest population (1141 birds) was observed in Lesser Whistling-duck Dendrocygna javanica followed 704 birds in Cattle Egret Bubulcus ibis and 584 birds in Common Coot Fulica atra. The lowest population (20 birds) was recorded in Yellow Bitten Ixobrychus sinensis (Table 2).

In Ngwe-tha reservoir (Site II), the numbers of waterbirds (2844 birds) were recorded. Bird numbers reached a peak in December (754 birds), January (875 birds) and February (833 birds) and gradually decline as birds left the reservoir in May (294 birds) and June (266 birds). The highest population were observed in Common Coot Fulica atra (817 birds) followed 473 birds in Little Cormorant Microcarbo niger and 365 birds in Lesser Whistling-duck Dendrocygna javanica while the lowest population was found in Pheasant-tailed Jacana Hydrophasianus chirurgus (four birds) (Table 3).

According to ANOVA test, the population of waterbird between Salingyi and Ngwe-tha reservoirs was significantly different 
$(\mathrm{F}(1,22)=11.118, \mathrm{p}=0.003)$. Thus Salingyi reservoir was higher number of waterbird population than Ngwe-tha. In Site I, the number of Lesser Whistling-duck Dendrocygna javanica was 1141 birds while in Site II was 365 birds. Common Coot Fulica atra was 584 birds in Site I, and 817 birds registered for the same species in Site II.
The Cattle Egret Bubulcus ibis was 584 birds in Site I while in Site II was 123 birds. Little Cormorant Microcarbo niger was 396 birds in Site I, and 473 birds recorded for the same species in Site II (Figure 2 ). This indicated that both reservoir habitats may vary in waterbird population.

Table I Status of the waterbird species recorded in different reservoirs June 2017 to May 2018

\begin{tabular}{|c|c|c|c|c|c|c|}
\hline Order & Family & Scientific name & Common name & IUCN Redlist & Status & Foraging groups \\
\hline & & Dendrocygna javanica & Lesser whistling-duck & LC & $\mathrm{R}$ & $\mathrm{Db}$ \\
\hline Anseriformormes & & Anas poecilorhyncha & Indian spot-billed duck & LC & $\mathrm{R}$ & $\mathrm{Db}$ \\
\hline \multirow{4}{*}{ Podicipediformes } & & Tachybaptus ruficollis & Little grebe & LC & $\mathrm{R}$ & $\mathrm{Di}$ \\
\hline & Podicipedidae & & & & & \\
\hline & & Podiceps cristatus & Great crested grebe & LC & $\mathrm{R}$ & $\mathrm{Di}$ \\
\hline & & Amaurornis phoenicurus & White-breasted waterhen & LC & $\mathrm{R}$ & $\mathrm{Sb}$ \\
\hline \multirow[t]{7}{*}{ Gruiformes } & Rallidae & Gallinula chloropus & Common morhen & LC & $\mathrm{R}$ & $\mathrm{Di}$ \\
\hline & & Fulica atra & Common coot & LC & WV & $\mathrm{Di}$ \\
\hline & & Plegadis falcinellus & Glossy ibis & LC & WV & $\mathrm{Sb}$ \\
\hline & & Ixobrychus sinensis & Yellow bitten & LC & $\mathrm{R}$ & $\mathrm{Sb}$ \\
\hline & & Ardeola grayii & Indian pond-heron & LC & $\mathrm{R}$ & $\mathrm{Sb}$ \\
\hline & & Ardeola bacchus & Chinese pond-heron & LC & $\mathrm{R}$ & $\mathrm{Sb}$ \\
\hline & & Bubulcus ibis & Cattle egret & LC & $\mathrm{R}$ & $\mathrm{Sb}$ \\
\hline \multicolumn{7}{|l|}{ Pelecaniformes } \\
\hline & & Ardea cinerea & Grey heron & LC & $\mathrm{R}$ & $\mathrm{Sb}$ \\
\hline & Arueruae & Ardea purpurea & Purple heron & LC & $\mathrm{R}$ & $\mathrm{Sb}$ \\
\hline & & Ardea alba & Great white egret & LC & $\mathrm{R}$ & $\mathrm{Sb}$ \\
\hline & & Egretta garzetta & Little egret & LC & $\mathrm{R}$ & $\mathrm{Sb}$ \\
\hline & & Microcarbo niger & Little cormorant & LC & $\mathrm{R}$ & $\mathrm{Di}$ \\
\hline & Phalacrocoracidae & & & & & \\
\hline \multirow[t]{5}{*}{ Suliformes } & & Phalacrocorax carbo & Great cormorant & LC & $\mathrm{R}$ & $\mathrm{Di}$ \\
\hline & Recurvirostidae & Himantopus himantopus & Black-winged stilt & LC & WV & $\mathrm{Sb}$ \\
\hline & & Charadrius dubius & Little ringed plover & LC & WV & $\mathrm{Sb}$ \\
\hline & Charadriidae & Vanellus cinereus & Grey-headed lapwing & LC & $\mathrm{R}$ & $\mathrm{Sb}$ \\
\hline & & Vanellus indicus & Red-wattled lapwing & LC & $\mathrm{R}$ & $\mathrm{Sb}$ \\
\hline \multirow[t]{4}{*}{ Charadriiformes } & Jacanidae & Hydrophasianus chirurgus & Pheasant-tailed jacana & LC & $\mathrm{R}$ & $\mathrm{Sb}$ \\
\hline & & Gallinago gallinago & Common snipe & LC & WV & $\mathrm{Sb}$ \\
\hline & Scolopacidae & Actitis hypoleucos & Common sandpiper & LC & WV & $\mathrm{Sb}$ \\
\hline & & Tringa glareola & Wood sandpiper & & WV & $\mathrm{Sb}$ \\
\hline
\end{tabular}

R, resident;WV, winter visitor; LC, least concern; NT, near threatened; Db, dabbling duck; Di, diving waterbird; Sb, shorebird 
Table 2 Monthly occurrence of waterbird population in Salingyi reservoir

\begin{tabular}{|c|c|c|c|c|c|c|c|c|c|c|c|c|c|c|}
\hline No & Scientific name & June & July & Aug & Sep & Oct & Nov & Dec & Jan & Feb & Mar & Apr & May & Total \\
\hline I & Dendrocygna javanica & 59 & 78 & 88 & 92 & 99 & 106 & 120 & 121 & 128 & 107 & 78 & 65 & || $4 \mid$ \\
\hline 2 & Tadorna ferruginea & 0 & 0 & 0 & 0 & 0 & 50 & 72 & 95 & 92 & 32 & 0 & 0 & 341 \\
\hline 3 & Anas poecilorhyncha & 21 & 12 & 22 & 24 & 28 & 28 & 26 & 28 & 32 & 21 & 22 & 18 & 282 \\
\hline 4 & Tachybaptus ruficollis & 14 & 15 & 18 & 20 & 28 & 30 & 28 & 34 & 23 & 22 & 18 & 12 & 262 \\
\hline 5 & Amaurornis phoenicurus & 0 & 14 & 18 & 10 & 14 & 10 & 12 & 18 & 0 & 0 & 0 & 0 & 96 \\
\hline 6 & Gallinula chloropus & 18 & 16 & 10 & 21 & 28 & 30 & 30 & 32 & 33 & 29 & 22 & 21 & 290 \\
\hline 7 & Fulica atra & 0 & 0 & 0 & 0 & 0 & 106 & 132 & 160 & 108 & 78 & 0 & 0 & 584 \\
\hline 8 & Threskiornis melanocephalus & 0 & 0 & 0 & 0 & 0 & 0 & 22 & 48 & 62 & 0 & 0 & 0 & 132 \\
\hline 9 & Plegadis falcinellus & 0 & 0 & 0 & 0 & 0 & 32 & 38 & 36 & 38 & 22 & 0 & 0 & 166 \\
\hline 10 & Ixobrychus sinensis & I & 2 & 2 & 2 & 2 & 2 & 2 & 2 & 2 & I & I & $\mathrm{I}$ & 20 \\
\hline 12 & Ardeola bacchus & 6 & 9 & 6 & 5 & 9 & 8 & 4 & 6 & 5 & 4 & 6 & 4 & 72 \\
\hline 13 & Bubulcus ibis & 49 & 50 & 28 & 44 & 46 & 54 & 68 & 70 & 68 & 79 & 82 & 66 & 704 \\
\hline 14 & Ardea cinerea & 5 & 4 & 4 & 6 & 8 & 5 & 8 & 7 & 12 & II & 9 & 6 & 85 \\
\hline 15 & Ardea purpurea & 2 & 2 & 3 & 3 & 4 & 3 & 4 & 4 & 3 & 4 & 2 & 3 & 37 \\
\hline 16 & Ardea alba & 5 & 3 & 6 & 10 & 8 & 10 & 8 & 8 & 12 & 8 & 6 & 4 & 88 \\
\hline 17 & Ardea intermedia & 19 & 14 & 10 & 20 & 20 & 20 & 18 & 22 & 21 & 20 & 18 & 15 & 217 \\
\hline 18 & Egretta garzetta & 23 & 38 & 34 & 52 & 40 & 48 & 30 & 36 & 32 & 36 & 29 & 22 & 420 \\
\hline 19 & Microcarbo niger & 16 & 18 & 14 & 21 & 37 & 42 & 46 & 42 & 41 & 49 & 38 & 32 & 396 \\
\hline 20 & Phalacrocorax carbo & 0 & 2 & 2 & 4 & 4 & 2 & 4 & 2 & 2 & 3 & 0 & 0 & 25 \\
\hline 21 & Himantopus himantopus & 0 & 0 & 0 & 0 & 0 & 4 & 6 & 6 & 10 & 8 & 0 & 0 & 34 \\
\hline 24 & Vanellus indicus & 6 & 9 & 10 & 8 & 6 & 6 & 6 & 4 & 8 & 9 & 8 & 7 & 87 \\
\hline 25 & Hydrophasianus chirurgus & 0 & 0 & 0 & 0 & 0 & 0 & 16 & 30 & 22 & 28 & 12 & 0 & 108 \\
\hline 26 & Gallinago gallinago & 0 & 0 & 0 & 0 & 0 & 0 & 12 & 16 & 18 & 12 & 0 & 0 & 58 \\
\hline 27 & Actitis hypoleucos & 0 & 0 & 0 & 0 & 0 & 0 & 8 & 6 & 8 & 4 & 0 & 0 & 26 \\
\hline \multirow[t]{3}{*}{28} & Tringa glareola & 0 & 0 & 0 & 0 & 0 & 0 & 12 & 8 & 10 & 8 & 0 & 0 & 38 \\
\hline & Total individuals & 266 & 297 & 288 & 358 & 400 & 626 & 754 & 875 & 833 & 630 & 367 & 294 & 5988 \\
\hline & Total species & 15 & 18 & 18 & 18 & 18 & 23 & 28 & 28 & 27 & 26 & 16 & 15 & \\
\hline
\end{tabular}

Table 3 Monthly occurrence of waterbird population in Ngwe-tha reservoir

\begin{tabular}{|c|c|c|c|c|c|c|c|c|c|c|c|c|c|c|}
\hline No & Scientific name & June & July & Aug & Sep & Oct & Nov & Dec & Jan & Feb & Mar & Apr & May & Total \\
\hline I & Dendrocygna javanica & 19 & 20 & 21 & 30 & 32 & 42 & 40 & 41 & 40 & 32 & 30 & 18 & 365 \\
\hline 2 & Tadorna ferruginea & 0 & 0 & 0 & 0 & 0 & 0 & 56 & 62 & 40 & 22 & 0 & 0 & 180 \\
\hline 3 & Anas poecilorhyncha & 6 & 4 & 4 & 6 & 6 & 12 & 8 & 14 & 12 & 6 & 10 & 8 & 96 \\
\hline 4 & Tachybaptus ruficollis & 6 & 6 & 3 & 4 & 5 & 6 & 3 & 4 & 12 & 10 & 8 & 6 & 73 \\
\hline 5 & Podiceps cristatus & 0 & 0 & 2 & 3 & 2 & I & 2 & 0 & 0 & 0 & 0 & 0 & 10 \\
\hline 6 & Amaurornis phoenicurus & 16 & 4 & 6 & 3 & 4 & 5 & 6 & 5 & 18 & 16 & 19 & 18 & 120 \\
\hline 7 & Gallinula chloropus & 8 & 9 & 14 & 16 & 22 & 24 & 20 & 22 & 18 & 14 & 12 & 8 & 187 \\
\hline 8 & Fulica atra & 0 & 0 & 0 & 0 & 0 & 86 & 138 & 201 & 212 & 180 & 0 & 0 & 817 \\
\hline 9 & Ixobrychus sinensis & I & I & 1 & 2 & 2 & 2 & 2 & 2 & 2 & 1 & 2 & 1 & 19 \\
\hline 10 & Ardeola grayii & 8 & 2 & 2 & 3 & I & 2 & 2 & 2 & 8 & 7 & 9 & 6 & 52 \\
\hline II & Ardeola bacchus & 2 & I & 2 & 2 & 3 & 2 & 2 & 3 & 2 & 3 & 2 & I & 25 \\
\hline
\end{tabular}




\begin{tabular}{|c|c|c|c|c|c|c|c|c|c|c|c|c|c|c|}
\hline No & Scientific name & June & July & Aug & Sep & Oct & Nov & Dec & Jan & Feb & Mar & Apr & May & Total \\
\hline 12 & Bubulcus ibis & 12 & 6 & 8 & 7 & 6 & 12 & 11 & 13 & 12 & 11 & 12 & 13 & 123 \\
\hline 13 & Ardea cinerea & 2 & 2 & 3 & 3 & 2 & 2 & 3 & 2 & 2 & I & 2 & 2 & 26 \\
\hline 14 & Ardea purpurea & 2 & 2 & I & 2 & 2 & 1 & 2 & 2 & 2 & 1 & 2 & 1 & 20 \\
\hline 15 & Ardea alba & 3 & 3 & 2 & 3 & 4 & 5 & 5 & 6 & 6 & 5 & 3 & 2 & 47 \\
\hline 16 & Ardea intermedia & 2 & 2 & 4 & 3 & 2 & 3 & 4 & 3 & 4 & 4 & 3 & 2 & 36 \\
\hline 17 & Egretta garzetta & 5 & 6 & 7 & 6 & 5 & 7 & 6 & 8 & 7 & 6 & 6 & 4 & 73 \\
\hline 18 & Microcarbo niger & 32 & 22 & 26 & 23 & 37 & 38 & 56 & 68 & 58 & 39 & 38 & 36 & 473 \\
\hline 19 & Phalacrocorax carbo & 0 & 2 & 4 & 2 & 2 & 12 & 13 & 12 & 8 & 6 & 4 & 0 & 65 \\
\hline 20 & Vanellus cinereus & 0 & 2 & 2 & 2 & 3 & 2 & 3 & 2 & 0 & 0 & 0 & 0 & 16 \\
\hline 21 & Vanellus indicus & 0 & 2 & 3 & 2 & 3 & 3 & 2 & 2 & 0 & 0 & 0 & 0 & 17 \\
\hline \multirow[t]{3}{*}{22} & Hydrophasianus chirurgus & 0 & 0 & 0 & 0 & 0 & 0 & 2 & 2 & 0 & 0 & 0 & 0 & 4 \\
\hline & Total individuals & 124 & 96 & 115 & 122 & 143 & 267 & 386 & 476 & 463 & 364 & 162 & 126 & 2844 \\
\hline & Total species & 15 & 18 & 19 & 19 & 19 & 20 & 22 & 21 & 18 & 18 & 16 & 15 & \\
\hline
\end{tabular}

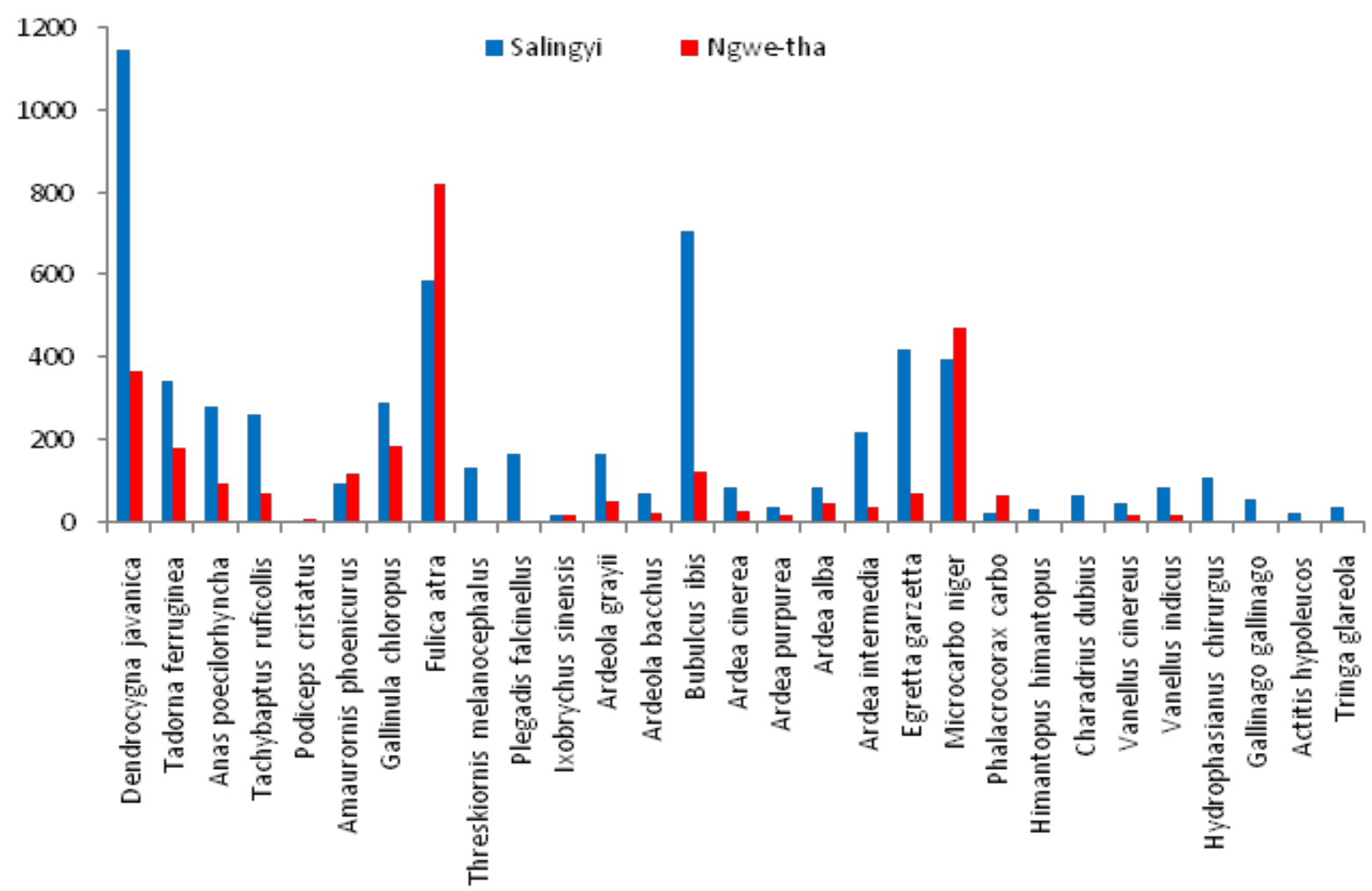

Figure 2 Comparison of waterbird population in two different reservoirs.

\section{Comparison of Population of waterbirds based on foraging behavior}

In the present study, the waterbird species were grouped based on foraging behavior and habitats use such as dabbling duck, diving and shorebird. In Salingyi reservoir, a total of 28 species of waterbirds, 20 species were shore birds ( $\mathrm{Sb}$ ), five species were diving birds (Di) and three species were dabbling ducks $(\mathrm{Db})$. The highest population of waterbirds recorded in shorebirds 2667 birds followed by dabbling ducks 1764 birds and diving birds 1557 birds. In Ngwe-tha reservoir, a total of 22 species of waterbirds were recorded and included 13 species of shore birds ( $\mathrm{Sb}$ ), six species of diving birds (Di) and three species of dabbling ducks $(\mathrm{Db})$. The highest population of waterbirds recorded also happened to be diving birds ( 1625 birds) followed by dabbling ducks (641 birds) and shorebirds (578 birds) (Table 4).

\section{Habitat utilization of waterbirds in different reservoirs}

Diving waterbirds and dabbling ducks were observed in open water body. They preferred open water body for foraging site. Cormorant species such as Microcarbo niger and Phalacrocorax 
carbo were often resting and drying their wings at the edge of open water area. Dabbling ducks such as Dendrocygna javanica, Tadorna ferruginea and Anas poecilorhyncha were frequently observed in the marshy swamp and at the edge of open water area for feeding and resting. Shorebirds species such as Amaurornis phoenicurus, Plegadis falcinellus, Ixobrychus sinensis, Threskiornis melanocephalus, Ardeola grayii, Ardeola bacchus, Bubulcus ibis, Ardea cinerea, Ardea purpurea, Ardea alba, Ardea intermedia, Egretta garzetta, Himantopus himantopus, Charadrius dubius, Vanellus cinereus, Vanellus indicus, Hydrophasianus chirurgus, Gallinago gallinago, Actitis hypoleucos and Tringa glareola were observed in marshy swamp. Moreover, most of shorebirds species foraged at the water edge but some were resting. Some species of shorebirds were observed in agricultural field, grass and shrub lands (Figure 2). Herons and egrets often utilized the tree and shrub at roosting sites (Table 5\&6).

Table 4 Waterbird population of foraging groups in different reservoirs

\begin{tabular}{|c|c|c|c|c|}
\hline Foraging group & Scientific name & $\begin{array}{l}\text { Salingyi } \\
\text { reservoir }\end{array}$ & $\begin{array}{l}\text { Ngwe-tha } \\
\text { reservoir }\end{array}$ & Total \\
\hline \multirow{4}{*}{ Dabbling duck } & Dendrocygnajavanica & || $4 \mid$ & 365 & 1506 \\
\hline & Tadorna ferruginea & 341 & 180 & 521 \\
\hline & \multirow[t]{2}{*}{ Anas poecilorhyncha } & 282 & 96 & 378 \\
\hline & & 1764 & 641 & 2405 \\
\hline \multirow{7}{*}{ Diving waterbird } & Tachybaptus ruficollis & 262 & 73 & 335 \\
\hline & Podiceps cristatus & 0 & 10 & 10 \\
\hline & Gallinula chloropus & 290 & 187 & 477 \\
\hline & Fulica atra & 584 & 817 & $|40|$ \\
\hline & Microcarbo niger & 396 & 473 & 869 \\
\hline & \multirow[t]{2}{*}{ Phalacrocorax carbo } & 25 & 65 & 90 \\
\hline & & 1557 & 1625 & 3182 \\
\hline \multirow{22}{*}{ Shorebird } & $\begin{array}{l}\text { Amaurornis } \\
\text { phoenicurus }\end{array}$ & 96 & 120 & 216 \\
\hline & Plegadis falcinellus & 166 & 0 & 166 \\
\hline & Ixobrychus sinensis & 20 & 19 & 39 \\
\hline & $\begin{array}{l}\text { Threskiornis } \\
\text { melanocephalus }\end{array}$ & 132 & 0 & 132 \\
\hline & Ardeola grayii & 165 & 52 & 217 \\
\hline & Ardeola bacchus & 72 & 25 & 97 \\
\hline & Bubulcus ibis & 704 & 123 & 827 \\
\hline & Ardea cinerea & 85 & 26 & III \\
\hline & Ardea purpurea & 37 & 20 & 57 \\
\hline & Ardea alba & 88 & 47 & 135 \\
\hline & Ardea intermedia & 217 & 36 & 253 \\
\hline & Egretta garzetta & 420 & 73 & 493 \\
\hline & $\begin{array}{l}\text { Himantopus } \\
\text { himantopus }\end{array}$ & 34 & 0 & 34 \\
\hline & Charadrius dubius & 68 & 0 & 68 \\
\hline & Vanellus cinereus & 46 & 16 & 62 \\
\hline & Vanellus indicus & 87 & 17 & 104 \\
\hline & $\begin{array}{l}\text { Hydrophasianus } \\
\text { chirurgus }\end{array}$ & 108 & 4 & 112 \\
\hline & Gallinago gallinago & 58 & 0 & 58 \\
\hline & Actitis hypoleucos & 26 & 0 & 26 \\
\hline & \multirow[t]{2}{*}{ Tringa glareola } & 38 & 0 & 38 \\
\hline & & 2667 & 578 & 3245 \\
\hline & Total & 5988 & 2844 & 8832 \\
\hline
\end{tabular}

Table 5 Occurrence of habitat types of waterbird species recorded in Salingyi reservoir

\begin{tabular}{|c|c|c|c|c|c|}
\hline \multirow{2}{*}{$\begin{array}{l}\text { Sr. } \\
\text { No. }\end{array}$} & \multirow{2}{*}{$\begin{array}{l}\text { Scientific } \\
\text { name }\end{array}$} & \multicolumn{4}{|c|}{ Habitat types } \\
\hline & & $\begin{array}{l}\text { Open } \\
\text { water body }\end{array}$ & $\begin{array}{l}\text { Marshy } \\
\text { swamp }\end{array}$ & $\begin{array}{l}\text { Agricultural } \\
\text { field }\end{array}$ & $\begin{array}{l}\text { Grass } \\
\text { and shrub } \\
\text { lands }\end{array}$ \\
\hline I & $\begin{array}{l}\text { Dendrocygnaja- } \\
\text { vanica }\end{array}$ & + & + & - & 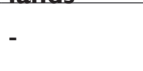 \\
\hline 2 & $\begin{array}{l}\text { Tadorna } \\
\text { ferruginea }\end{array}$ & + & + & - & - \\
\hline 3 & $\begin{array}{l}\text { Anas } \\
\text { poecilorhyncha }\end{array}$ & + & + & - & - \\
\hline 4 & $\begin{array}{l}\text { Tachybaptus } \\
\text { ruficollis }\end{array}$ & + & - & - & - \\
\hline 5 & $\begin{array}{l}\text { Amaurornis } \\
\text { phoenicurus }\end{array}$ & - & + & - & + \\
\hline 6 & $\begin{array}{l}\text { Gallinula } \\
\text { chloropus }\end{array}$ & + & - & - & + \\
\hline 7 & Fulica atra & + & - & - & - \\
\hline 8 & $\begin{array}{l}\text { Threskiornis } \\
\text { melanocephalus }\end{array}$ & - & + & - & - \\
\hline 9 & $\begin{array}{l}\text { Plegadis } \\
\text { falcinellus }\end{array}$ & - & + & - & + \\
\hline 10 & $\begin{array}{l}\text { Ixobrychus } \\
\text { sinensis }\end{array}$ & - & + & - & - \\
\hline 11 & Ardeola grayii & - & + & + & + \\
\hline 12 & Ardeola bacchus & - & + & + & + \\
\hline 13 & Bubulcus ibis & - & + & + & + \\
\hline 14 & Ardea cinerea & - & + & + & + \\
\hline 15 & Ardea purpurea & - & + & + & + \\
\hline 16 & Ardea alba & - & + & + & + \\
\hline 17 & $\begin{array}{l}\text { Ardea } \\
\text { intermedia }\end{array}$ & - & + & + & + \\
\hline 18 & Egretta garzetta & - & + & + & + \\
\hline 19 & $\begin{array}{l}\text { Microcarbo } \\
\text { niger }\end{array}$ & + & - & - & - \\
\hline 20 & $\begin{array}{l}\text { Phalacrocorax } \\
\text { carbo }\end{array}$ & + & - & - & - \\
\hline 21 & $\begin{array}{l}\text { Himantopus } \\
\text { himantopus }\end{array}$ & - & + & - & - \\
\hline 22 & $\begin{array}{l}\text { Charadrius } \\
\text { dubius }\end{array}$ & - & + & + & - \\
\hline 23 & $\begin{array}{l}\text { Vanellus } \\
\text { cinereus }\end{array}$ & - & + & - & - \\
\hline 24 & Vanellus indicus & - & + & - & - \\
\hline 25 & $\begin{array}{l}\text { Hydrophasianus } \\
\text { chirurgus }\end{array}$ & - & + & - & - \\
\hline 26 & $\begin{array}{l}\text { Gallinago } \\
\text { gallinago }\end{array}$ & - & + & + & - \\
\hline 27 & $\begin{array}{l}\text { Actitis } \\
\text { hypoleucos }\end{array}$ & - & + & + & - \\
\hline 28 & Tringa glareola & - & + & + & - \\
\hline Total & & 8 & 23 & 12 & 11 \\
\hline
\end{tabular}

Present (+),Absent (-) 
Table 6 Occurrence of habitat types of waterbird species recorded in Ngwetha reservoir

\begin{tabular}{|c|c|c|c|}
\hline \multirow[b]{2}{*}{ No } & \multirow[b]{2}{*}{ Scientific name } & \multicolumn{2}{|c|}{ Habitat types } \\
\hline & & $\begin{array}{l}\text { Open } \\
\text { water body }\end{array}$ & $\begin{array}{l}\text { Edge of open } \\
\text { water area }\end{array}$ \\
\hline I & Dendrocygnajavanica & + & + \\
\hline 2 & Tadorna ferruginea & + & + \\
\hline 3 & Anas poecilorhyncha & + & + \\
\hline 4 & Tachybaptus ruficollis & + & - \\
\hline 5 & Podiceps cristatus & + & - \\
\hline 6 & Amaurornis phoenicurus & - & + \\
\hline 7 & Gallinula chloropus & + & - \\
\hline 8 & Fulica atra & + & - \\
\hline 9 & Ixobrychus sinensis & - & + \\
\hline 10 & Ardeola grayii & - & + \\
\hline II & Ardeola bacchus & - & + \\
\hline 12 & Bubulcus ibis & - & + \\
\hline 13 & Ardea cinerea & - & + \\
\hline 14 & Ardea purpurea & - & + \\
\hline 15 & Ardea alba & - & + \\
\hline 16 & Ardea intermedia & - & + \\
\hline 17 & Egretta garzetta & - & + \\
\hline 18 & Microcarbo niger & + & + \\
\hline 19 & Phalacrocorax carbo & + & + \\
\hline 20 & Vanellus cinereus & - & + \\
\hline 21 & Vanellus indicus & - & + \\
\hline 22 & $\begin{array}{l}\text { Hydrophasianus } \\
\text { chirurgus }\end{array}$ & - & + \\
\hline Total & & 9 & 18 \\
\hline
\end{tabular}

Present (+), Absent (-)

\section{Discussion}

In this study, a total of 8832 individuals of 29 waterbird species were recorded from two different reservoirs namely, Salingyi and Ngwe-tha reservoir at Sagaing Region. In Salingyi reservoir, the 5988 individuals of 28 species were recorded and the 2844 individuals of 22 species were in Ngwe-tha reservoir during the study period. The individual of waterbirds in Salingyi reservoir were more observed than in Ngwe-tha reservoir. These differences are due to the habitat conditions of two reservoirs where Salingyi reservoir has more diverse habitat compared to Ngwe-tha reservoir. Salingyi reservoir composed of open water area, shallow water area, marshy swamp, damp soil, flooded agricultural fields, cultivated fields, shrub and bushy area, small grass land and small trees around the study area. Ngwe-tha reservoir composed of a wide open water area and edge of open water area. Myo Sandar Win ${ }^{14}$ stated that the bird abundance is directly related to the habitat types and conditions. The distribution and abundance of many bird species are determined by the composition of the vegetation that comprises a major element of their habitats. ${ }^{15}$

In present study, among 29 species, 9 species were winter visitor and 20 species were residents at Salingyi and Ngwe-tha reservoir in Salingyi Township, Sagaing Region. Htay Khaing ${ }^{16}$ recorded that 12 species were winter visitors, 19 species were residents at Inmagyi wetland in Myinmu Township, Sagaing Region. Hla Toe ${ }^{17}$ revealed that nine species were winter visitors and 24 species were residents at Sunye In in Sintkaing Township, Mandalay Region. Nwet Nwet $\mathrm{Win}^{18}$ described that 13 species were winter visitors and 23 species were residents at Pauk In in Pakokku Township, Magway Region. These differences are due to the local rainfall, wetland area, wetland depth and major waterbird food-bases as the most important factors. Bhushan et al. ${ }^{19}$ described that many migratory birds from Northern hemisphere fly to the Southern every winter season to avoid the severe cold and scanty of food in the Northern part in the winter. They migrated into many Asian countries including Myanmar and assumed that this area is good habitat and available of food during their stay.

In present study, the highest number of bird was mostly observed in cold months (December, January and February) in both reservoirs. It is due to the migratory birds and local visitor birds are arrived in these reservoirs for hibernating and foraging. Especially migratory birds, Common Coot (Fulica atra) was recorded as higher number in cold months while the reduce number of these bird was found in hot season. The higher number of common coot was observed in Ngwetha reservoir than Salingyi reservoir because the total water surface area of Ngwe-tha reservoir is larger than Salingyi reservoir.

Holm et al. ${ }^{20}$ reported that Common Coots were observed in deeperwater areas and large surface area of the lagoon during November compared to October, and foraging by diving increased significantly. Khin Hnin Thet ${ }^{21}$ stated that Lesser whistling Duck Dendrocygna javanica showed increasing population of waterbirds throughout study period at Monywa Township Sagaing Region. Tin Htar Yee ${ }^{22}$ described that Lesser whistling Duck Dendrocygna javanica showed the highest population of waterbirds and very common species at Nyaung Kaing In (Lake), Monywa Township, Sagaing Region. In the present study, Lesser whistling Duck Dendrocygna javanica showed as the highest population in Salingyi reservoirs, is highly productive and good foraging sites for this species.

Bitterns and herons also preferred scattered emergent vegetation especially along the water body edges for foraging. This could be that emergent vegetation in shallow water provided suitable breeding and foraging habitat for a variety of aquatic animals such as fishes, amphibians and invertebrates which is easy to catch in shallow water due to low water depth. ${ }^{23}$ In the present study, the highest number of foraging groups of waterbirds was recorded in shore birds followed by diving birds and dabbling ducks in Salingyi reservoir. These result supported that the shallow water and marsh swamp of Salingyi reservoir provide favourable water level and good foraging habitat for a greater density and abundance of shore birds.

Nolet et al., ${ }^{24}$ described that higher water levels are also adverse to foraging efficiency of waterbirds. Deep wetlands supported diving birds and dabbling ducks, which were ubiquitous. In the present study, the diving waterbirds such as Little Grebe, Common Morhen, Common Coot, Little Cormorant and Great Cormorant preferred open water habitat of both reservoirs for foraging sites. Chan-Woo et al., revealed that diving birds were abundant in higher water level. Diving waterbirds feed in deeper water depth than other waterbirds. The present study indicated that waterbird populations were directly influenced by the availability of foraging habitats and have preference to wetland habitat with surface water that supply suitable prey organisms for foraging. Moreover, the population of waterbird species was found to be associated with the water level and the availability of the food resource changes in different habitats of reservoirs.

\section{Conclusion}

The present study indicated that Salingyi reservoir supported higher waterbird population as compared to Ngwe-tha reservoir. This is due to richness of habitat types (open water body, marshy swamp, 
agricultural field, grass and shrub lands), availability of abundance food sources (invertebrate, vegetable matter, fishes, amphibians, reptiles and small mammals) and also shelter from predators. The population of waterbird species was found to be associated with the water level and the availability of the food resource changes in different habitats.

\section{Acknowledgements}

I am very grateful to Dr. Thura Oo, Rector, Dr. Sein Sein Aung and Dr. Thet Naing Oo, Prorectors, Monywa University for their permission to carry out the present research. I would like to thank Dr Khin Soe Win, Professor and Head, Department of Zoology, Monywa University for her encouragement and providing facilities for the research in the Department.

\section{Conflicts of interest}

Author declares that there are no conflicts of interest.

\section{Funding}

None.

\section{References}

1. Kumar P, Gupta SK. Diversity and abundance of wetland birds around Kurukshetra, India. Our Nature. 2009;7(1):187-192.

2. Ramsar Burea Convention. The Ramsar list of wetlands of international importance. The Ramsar conventation of wetland. 2010.

3. Beury JN, Baker DS, Huggins DG. Wetlands in three ecoregion of the central plains. Kansas Biological Survey Report. 2008;

4. Buckton S. Managing wetlands for sustainable livelihoods at Koshi Tappu. Danphe. 2007;16:12-13.

5. Rajpar MN, Zakaria M. Effects of water level fluctuation on waterbirds distribution and aquatic vegetation composition at Natural Wetland Reserve, Peninsular Malaysia. ISRN Ecology. 2011;1-13.

6. Davies J, Sebastian AC, Chan S. A wetland inventory for Myanmar. Thailand: Durnsutha Press Company Ltd; 2004; p. 591.

7. Zakaria M, Rajpar MN. Density and Diversity of Water Birds and Terrestrial birds in Man-made Marsh, Malaysia. Sains Malaysiana. 2013;42(10):1483-1492.

8. Isola CR, Colwell MA, Taft OW, et al. Interspecific differences in habitat use of shorebirds and waterfowl foraging in managed wetlands of California's San Joaquin Valley. Waterbirds. 2002;23(2):196-203.

9. King BE, Dickinson EC. Collins Field Guide to the Birds of Southeast Asia. London: Harpet Collins publishers; 1975; p. 480.
10. Smythies BE. The Birds of Burma. London: Oliver and Boyd Ltd; 2001; p. 432.

11. Robson C. Helm field guid, Birds of South-East Asia. $2^{\text {nd }}$ edn. London: Bloomsbury Publishing; 2015. p.544

12. BirdLife International Web Site. Birdlife International: 2015 The Bird Life checklist of the birds of the world, with conservation status and taxonomic sources.

13. Chan-Woo L, Kim GY, Jang JD, et al. Water Level Fluctuation and Habitat Use Pattern of Wintering Waterbirds in The Junam Reservoir Area, South Korea. Journal Biosains. 2006;17(2):79-92.

14. Myo Sandar Win. Population fluctuation and habitat utilization of migratory birds in Indawgi Wetlands Bird Sanctuary. Myanmar: PhD Dissertation, University of Yangon; 2008.

15. Lee P, Rotenberry JT. Relationships between bird species and tree species assemblages in forested habitats of eastern North America. Journal of Biogeography. 2005;32(7):1139-1150.

16. Htay Khaing. Waterbird communities at Inmagyi wetland in Myinmu Township, Sagaing Region. Myanmar: PhD Dissertation, Department of Zoology, University of Mandalay; 2017.

17. Hla Toe. Seasonal abundance and diversity of waterbirds at Sunye In (Lake) with special emphasis on some aspects of breeding ecology of Porphyrio poliocephalus Latham, 1801. Myanmar: PhD Dissertation, University of Mandalay; 2012.

18. Nwet Nwet Win. Seasonal abundance and species diversity of waterbirds at Pauk In (Lake), Magway Region with emphasis on breeding ecology of Spot-billed duck, Anas poecilorhyncha Forster, 1781. Myanmar: PhD Dissertation, University of Mandalay; 2012

19. Bhushan B, Graham F, Akria H, et al. A Field Guide to the Waterbirds of Asia, Tokyo: Wild Bird Society of Japan; 1993.

20. Holm TE, Laursen K, Clausen P. The feeding ecology and distribution of Common Coots Fulica by hunting taking place in adjacent areas. Bird Study. 2011;58(3):321-329.

21. Khin Hnin Thet. Ecological aspects of avian fauna around Monywa environs, Sagaing Region. Myanmar: PhD Dissertation, University of Mandalay; 2013.

22. Tin Htar Yee. Habitat utilizations of waterbirds at Nyaung Kaing In (Lake), Monywa Township. Myanmar: M.Sc Thesis, Department of Zoology, University of Monywa; 2017.

23. Rajpar MN, Zakaria M. Avian density in different habitat types at Paya Indah Natural Wetland Reserve, Penninsular Malaysia. The Journal of Animal and Plant Sciences. 2013;23(4):1019-1033.

24. Nolet BA, Fuld VN, Van Rijswijk MEC. Foraging costs and accessibility as determinants of giving-up densities in a swan-pondweed system. Oikos. 2006;112(2):353-362. 